\title{
Pulmonary Nontuberculous Mycobacterial Infection in Infants: A Systematic Review
}

\author{
Alice $\mathrm{Bai}^{\prime}$ \\ Olivia Belda' \\ Amrita Dosanjh $\mathbb{1}^{2}$ \\ 'Brown University, Providence, RI, USA; \\ ${ }^{2}$ Pediatric Respiratory, Affiliated Staff \\ Member, Department of Pediatrics, Rady \\ Children's Hospital, San Diego, CA, USA
}

Correspondence: Alice Bai

Brown University, 69 Brown St. Mail\#

5288, Providence, RI, 02912, USA

Tel +l 770 843-2062

Email alice_bai@brown.edu

\begin{abstract}
Limited information and literature exist examining pulmonary infections caused by nontuberculous mycobacterial specifically in an infant population. The objective of our study was to summarize clinical characteristics and outcomes of infant patients with nontuberculous mycobacterial pulmonary infection via systematic literature review to identify common diagnostic and treatment regimens for this infection in infants. A search of MEDLINE and PubMed databases in October 2019 using MeSH search terms "infant," "NTM," "pulmonary," and "Mycobacterium abscessus" yielded 139 articles. Inclusion criteria were i) English-language studies including cases and case series with ii) established nontuberculous mycobacterial pulmonary infection in iii) a patient population of infants no older than 24 months. Patients with cystic fibrosis and any study which did not contain relevant information such as infection and age were excluded. This yielded data on 37 patients extracted from 28 studies analyzed. The most common strain was Mycobacterium avium complex, isolated in $56.8 \%$ of patient diagnoses. Bronchoscopy/thoracoscopy with a subsequent culture were the most common diagnostic techniques, utilized in $64.9 \%$ of cases. Drug therapeutic treatment was utilized in $86 \%$ of cases, with a median of three drugs administered. Notable limitations of this study are the small sample size and its retrospective nature, which relies on information reported in previous case studies. Although there is limited formal clinician consensus on the treatment of NTM pulmonary infection and how it may differ in an infant population, our findings indicate an informal consensus typically involving diagnostic lung specimen culture and antibiotic therapy.
\end{abstract}

Keywords: nontuberculous mycobacterial, NTM, pulmonology, pediatrics

\section{Introduction}

Nontuberculous mycobacteria (NTM) are found commonly in the daily environment, notably in soil, water, and dust. Over 160 species of NTM have been identified, but infection and disease are rare relative to the ubiquity of these bacteria, although the likelihood of disease is increased in cases of underlying lung disease or immunodeficiency. ${ }^{1}$ While infected patients may remain asymptomatic, NTM disease can also be fatal even in immunocompetent patients; recent trends have shown an increase in NTM cases and mortality globally. ${ }^{2,3}$ NTM pulmonary disease is commonly identified in adults with previous lung disease, whereas in pediatric populations these infections occur most often in individuals with no previous lung disease. Immunodeficiencies and underlying pulmonary disease, commonly cystic fibrosis, increase risk for NTM pulmonary infection in children. ${ }^{4}$

Pulmonary NTM disease is frequently misdiagnosed as M. tuberculosis infection, due to the presence of similar symptoms in both cases, which can result in 
delayed diagnosis and proper treatment of patients. ${ }^{5}$ This challenge is especially pertinent in countries with high endemic tuberculosis rates, where testing materials for NTM are typically not readily available. ${ }^{6}$ Notably, industrialized countries report higher incidence of NTM infections than of tuberculosis, although the incidence specifically in pediatric populations is unclear due to lack of mandatory disease reporting. Studies of NTM prevalence in the larger population give results for mean age of infection far higher than childhood, typically ranging between 50 and 60 years old. ${ }^{7-11}$ Diagnosis is particularly challenging in young children, where following ATS/ IDSA can often result in missed diagnoses. ${ }^{4}$ Treatment of NTM pulmonary infections in the general population is also a source of controversy given a lack of data combined with extensive diversity of expert opinion. ${ }^{12}$

In this study, we systematically investigate the current literature for data regarding diagnosis and treatment of NTM in infants. Relatively little work exists focused on the diagnosis and treatment of NTM disease in infants, thus the purpose of the present study is to provide a preliminary synthesis of descriptive clinical, microbiological, and treatment information specific to infants with pulmonary NTM infection to diversify considerations about NTM pulmonary treatment by highlighting existing data on individual cases.

\section{Methods}

A literature search was conducted in October 2019 in MEDLINE and PubMed databases according to Preferred Reporting Items for Systematic Reviews and MetaAnalyses guidelines using the MeSH searches a) infant and NTM and pulmonary; b) Mycobacterium abscessus and infant and pulmonary; and c) Mycobacterium abscessus and infant. An additional 5 articles were added from a secondary search, yielding a total of 139 articles. No records were added from other sources. After removing duplicates, 118 studies remained. None of these studies were excluded from the screening stage; all full-text studies were assessed for eligibility.

Cases were only included if they met all inclusion criteria for this study: i) English-language studies including cases and case series with ii) established nontuberculous mycobacterial pulmonary infection in iii) a patient population of infants no older than 24 months. Patients with cystic fibrosis and any study which did not contain relevant information such as infection and age were excluded. Infant is standard terminology for this age range, and cases were additionally checked to ensure adherence to the age cutoff. Because patients with cystic fibrosis are known to be at increased risk for NTM pulmonary infection due to their underlying lung disease, we excluded these patients.

Diagnosis of pulmonary NTM infection was based on positive lung culture. Updated ATS/IDSA guidelines state that two positive sputum samples or one positive bronchial lavage should be used for diagnosis. ${ }^{13}$ Collection of two separate airway samples is not usually feasible for an infant population; we therefore accepted one sample and reported findings based on one lung procedure and a positive culture.

Articles were not limited to a specific date range. Articles were reviewed independently by two reviewers ( $\mathrm{AB}$ and $\mathrm{OB}$ ). Cases of dispute were resolved by discussion with a third reviewer (AD). Data was extracted from included articles by one reviewer (AB). Original source data was confirmed after analysis was completed. The review was not registered, and a protocol was not prepared. Data extracted from studies and used for analyses are presented in the Supplemental Materials ("Data Extraction" File).

\section{Statistical Analysis}

Descriptive data were extracted from included articles to a spreadsheet and analyzed using Google Sheets software. The data extracted were: gender; age at diagnosis; NTM type; treatment duration; drug provided; patient outcomes; immunocompetence; and diagnostic testing information including test type, acid-fast bacilli testing, and tuberculosis testing. Analysis pooled this data to find descriptive values, which were sums, percent, mean, and median \pm standard deviation as applicable. No data reported was assumed in our data to mean that no significant information was present in the case. A p value of $<0.05$ was considered significant.

\section{Results}

Abstracts and full-text articles were reviewed, and 90 studies were excluded per exclusion criteria, yielding 28 articles examining 37 total patients. Figure 1 summarizes the search procedures. Common reasons for exclusion were studies that presented only aggregate data, data on patients older than 24 months, and data on patients without pulmonary infection due to NTM. 


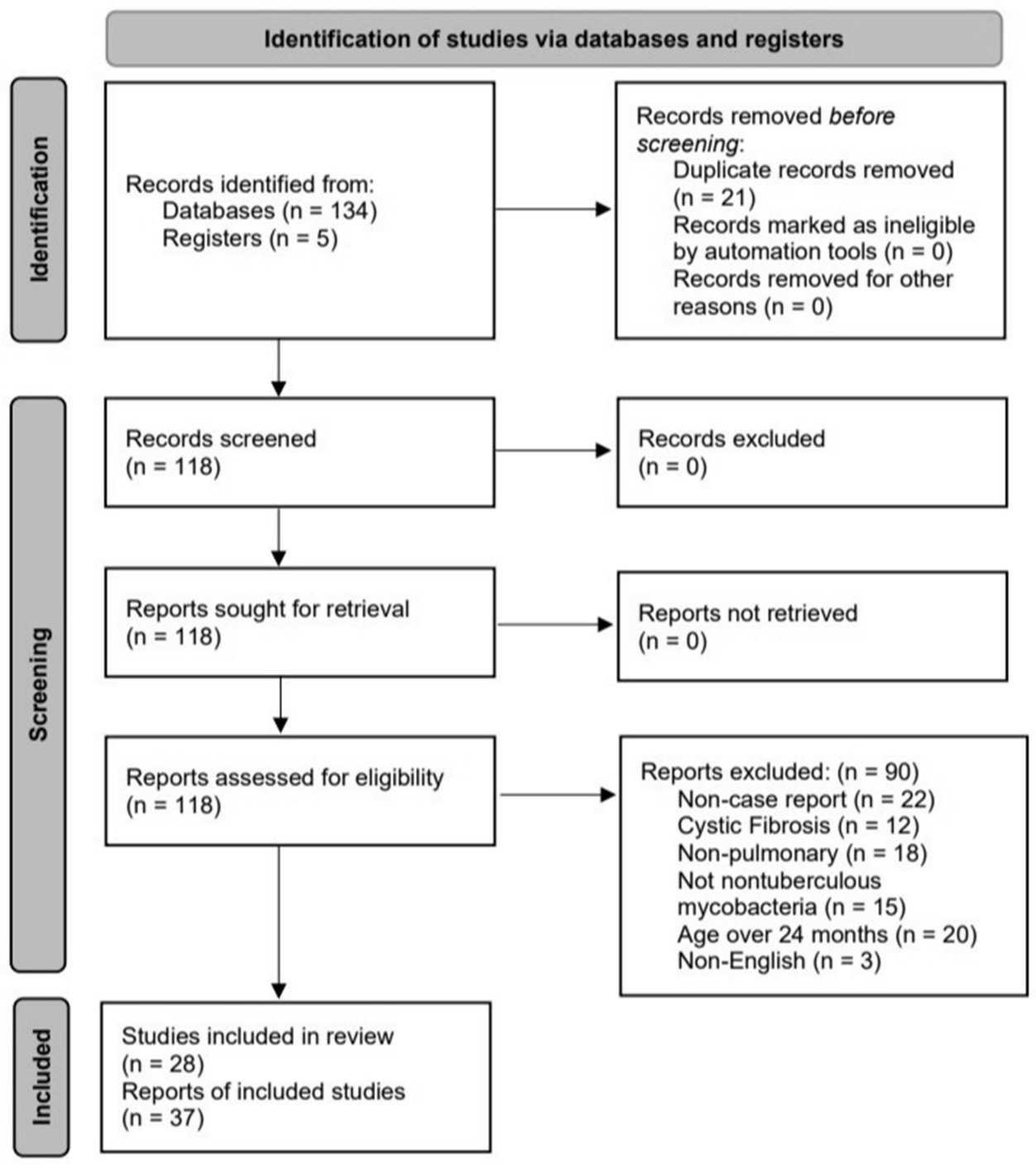

Figure I PRISMA flow-chart of article selection.

\section{Demographic Data}

Our study examined case reports from 37 patient cases. Demographic data are summarized in Table 1. The two patients who died were due to worsening pulmonary consolidations, and persistent diarrhoea and septicemia.

\section{Testing}

Diagnosis of NTM generally involved multiple forms of testing, including testing for $M$. tuberculosis infection. Case reports that did not mention testing were assumed to not have conducted that test. The most common procedure was the collection of sample material from bronchoscopy or thoracoscopy, with 24 of 37 patients undergoing this procedure. The two other most common sources for material for
NTM culture were bronchoalveolar lavage and gastric aspirate samples, with 16 and 11 patients undergoing these procedures respectively. Less commonly used sources for testing included 5 cases using PCR testing to identify the strain of NTM, 1 case using Niacin testing, and 1 use of Paranitrobenzoic acid testing.

In addition to seeking to isolate a specific strain of NTM, these tests were also used to identify the presence of acid-fast bacilli (AFB) in samples, which was noted in 32 cases. Samples from 21 patients $(56.8 \%)$ returned positive for AFB while 10 were negative $(27.0 \%)$, and 1 case discussed testing for AFB but not the results of these cultures. The remaining 6 did not mention AFB testing at all, resulting in $16.2 \%$ of cases with unknown AFB presence. 
Table I Demographics of Selected Study Population

\begin{tabular}{|l|l|l|}
\hline Demographics & $\mathbf{N}(\%)$ & \\
\hline Age at Diagnosis & & Median (IQR) \\
\hline$<6$ months & $9(24.3)$ & 3 months (I.9-4 months) \\
\hline 6 months - <12 months & $5(13.5)$ & 9 months (8-10 months) \\
\hline 12 months - <18 months & $13(35.1)$ & 13 months (I2-14 months) \\
\hline$\geq 18$ months & $10(27.0)$ & 22 months (20-23 months) \\
\hline Total & 37 & 12 months (6-18 months) \\
\hline Patient Sex & & \\
\hline Female & $20(54.1)$ & \\
\hline Male & $14(37.8)$ & \\
\hline Unspecified & $3(8.1)$ & \\
\hline Immunodeficiency & & \\
\hline Yes & $3(8.1)$ & \\
\hline No & $34(91.9)$ & \\
\hline Deaths & $2(5.4)$ & \\
\hline Yes & $33.2)$ & \\
\hline No & & \\
\hline Unknown & & \\
\hline & & \\
\hline
\end{tabular}

The majority of these tests were conducted on samples drawn from BAL and/or gastric aspirate sources. Examining the difference between these two major culture sources, BAL was used on 16 patients, with 10 of these cultures testing positive for AFB. Gastric aspirate samples were taken from 11 patients, with 5 of these samples returning positive for AFB. Figure 2 illustrates how many cases tested positive and negative for AFB from a given culture source: BAL culture results were positive for AFB $62.5 \%$ of the time, while gastric aspirate culture results were positive $45.5 \%$ of the time. Note that cases using BAL and gastric aspirate are not exclusive of the other.

At least one form of testing for M. tuberculosis was reported in 22 patients. These methods were the Tuberculin Skin Test (TST), Nucleic Acid Amplification Test (NAAT), QuantiFERON-TB Gold (QFT), and/or Interferon-Gamma Release Assay (IGRA). TST was used in all 22 of these cases, with 9 tests resulting in positive measurements. The mean induration measurement of these positive cases was $12.8 \mathrm{~mm} \pm 4.8 \mathrm{~mm}$. The more infrequently used tests resulted in 3 negative measures using IGRA, 2 negative measures using QFT, and one negative measure using NAAT.

MAC cases comprised the majority of cases where $M$. tuberculosis testing was also performed with TST. Of the 18 cases tested via TST, 10 were considered to be negative, while the other 8 tested positive.

The results of this analysis demonstrate potential difficulties in identifying and diagnosing NTM infection, given

\section{Acid-fast Bacilli Testing Results}

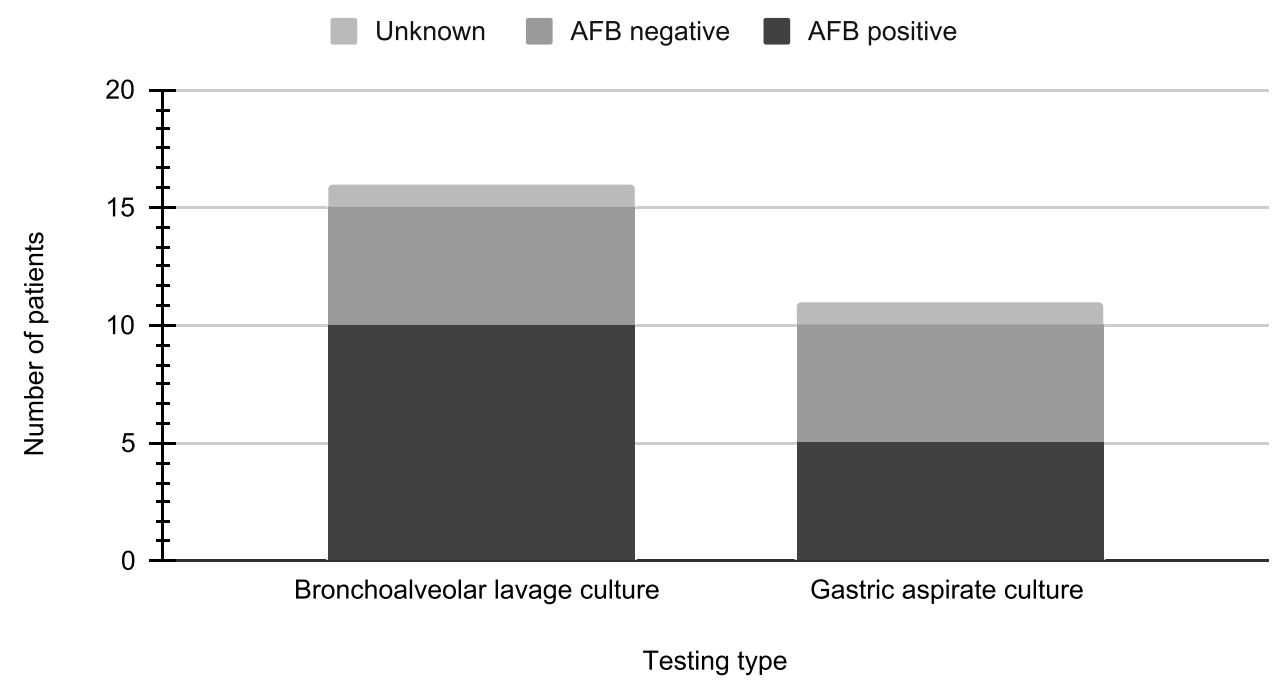

Figure 2 Results from AFB testing for two major sources of culture samples. 
that cases may test positive for M. tuberculosis, a much more common disease, via common testing methods such as TST.

\section{NTM Type}

There were a total of 6 NTM strains identified in patients, while one patient was also diagnosed with an unspecified mycobacterial strain. The types were Mycobacterium abscessus, M. avium/M. avium complex (MAC), M. chelonae, M. fortuitum, M. genavense, M. smegmatis. The most common NTM strain was $M$. avium and $M$. avium complex, identified in 21 patients $(56.8 \%)$. The next most common strains were $M$. abscessus and M. fortuitum, with 6 and 5 cases respectively. These results are included in Table 2.

\section{Type of Drugs Provided and Amount Administered}

A mean of $3.11 \pm 2.12$ drugs were administered per patient, and a median of 3 drugs were used. Five patients did not receive drugs for treatment following their diagnosis. A complete listing of drugs and the respective number of cases of use is as follows:

Amikacin (10), Azithromycin (8), Cefmetazole (1), Cefoxitin (3), Cefprozil (1), Ciprofloxacin (4), Clarithromycin (15), Clofazimine (2), Ethambutol (12), Ethionamide (1), Glucocorticoids (1), IFN-gamma (1), Imipenem (1), Isoniazid (7), Levofloxacin (1), Linezolid
Table 2 Frequency of Diagnosis of NTM Strain Species

\begin{tabular}{|l|r|r|}
\hline NTM Type & Number of Patients & Percentage \\
\hline M. abscessus & 6 & 16.2 \\
\hline M. avium complex (MAC) & 21 & 56.8 \\
\hline M. chelonae & 3 & 8.1 \\
\hline M. fortuitum & 5 & 13.5 \\
\hline M. genavense & 1 & 2.7 \\
\hline $\begin{array}{l}\text { M. smegmatis } \\
\text { Unspecified } \\
\text { mycobacterium }\end{array}$ & 1 & 2.7 \\
\hline
\end{tabular}

Note: Genus is abbreviated and each strain's name is italicized.

(2), Meropenem (2), Moxifloxacin (3), Netilmicin (1), Ofloxacin (1), PAS (para-aminosalicylic acid) (1), Prednisolone (1), Prednisone (2), Pyrazinamide (3), Rifabutin (4), Rifampicin (Rifampin) (18), Streptomycin (1), Trimethoprim-sulfamethoxazole (TMP/SMX) (2).

Table 3 illustrates the relationship between the most common drugs and their usage among specific strains of NTM. Among all NTM types, Rifampicin and Clarithromycin were most commonly used, but when comparing the percentage use of each drug per strain, we can see that for M. abcessus, Clarithromycin and Amikacin were most common. In

Table 3 Number of Patients Diagnosed with Strain Type and Percent Use of Medication per Strain

\begin{tabular}{|c|c|c|c|c|}
\hline Type vs Drug & M. abscessus & M. avium Complex & M. fortuitum & M. chelonae \\
\hline Rifampicin & $2(33 \%)$ & 12 (57\%) & I (20\%) & I (50\%) \\
\hline Clarithromycin & $4(66 \%)$ & $8(38 \%)$ & I (20\%) & $2(100 \%)$ \\
\hline Ethambutol & I (I7\%) & $9(43 \%)$ & - & I (50\%) \\
\hline Amikacin & $3(50 \%)$ & $3(14 \%)$ & $3(60 \%)$ & - \\
\hline Azithromycin & I (I7\%) & $4(19 \%)$ & I (20\%) & - \\
\hline Isoniazid & I (I7\%) & $6(29 \%)$ & - & - \\
\hline Ciprofloxacin & I (I7\%) & $2(10 \%)$ & I (20\%) & - \\
\hline Rifabutin & - & $4(19 \%)$ & - & - \\
\hline Moxifloxacin & I (I7\%) & I (5\%) & - & - \\
\hline Cefoxitin & $2(33 \%)$ & - & I $(20 \%)$ & - \\
\hline Pyrazinamide & - & $2(10 \%)$ & - & - \\
\hline Ofloxacin & - & - & - & I (50\%) \\
\hline Total cases & 6 & 21 & 5 & 2 \\
\hline
\end{tabular}

Note: Genus is abbreviated and each strain's name is italicized. 
M. avium, the three most common drugs were Rifampicin, Ethambutol, and Clarithromycin. Finally, $60 \%$ of M. fortuitum patients were administered the drug Amikacin. In addition, almost all of the drugs were used for more than one strain type, with the exception of Rifabutin and Pyrazinamide, which were used only for M. avium strain, and Ofloxacin, which was only administered in cases of $M$. chelonae.

\section{Discussion}

Data from the cases we examined indicate that mycobacteria in $M$. avium complex were the most common cause of pulmonary NTM infection in infants and that Rifampicin, Clarithromycin, Ethambutol, and Amikacin were the most frequently prescribed antibiotics used in the treatment of NTM infection. Additionally, our findings indicate that direct sample for culture via bronchoscopy or thoracoscopy was the most frequently used diagnostic procedure.

Our study's findings regarding specifically infants are in line with existing literature that cites MAC, which is comprised of various mycobacterial species such as $M$. intracellulare, $M$. avium, and $M$. chimaera, as the most common cause of pulmonary NTM infection. ${ }^{14,15}$ We also identified that the most commonly prescribed drugs were Rifampicin, Clarithromycin and Azithromycin (which is part of a macrolide treatment), Ethambutol, and Amikacin. Many other studies indicate that these are the most effective and common drugs for this infection: recommended treatment for MAC involves a triple-drug therapy of a macrolide (clarithromycin or azithromycin), ethambutol, and rifampicin, with additional amikacin or streptomycin prescribed in some severe cases. ${ }^{15-18}$ Cases treated with these drugs had fewer indices of recurrences, and in a Zimmerman study on infections in children, the adjusted mean cure rate was $73.1 \%$ with these drugs. $^{15,19}$

To our knowledge, our study is the first to systematically examine NTM pulmonary infection in an infant population. Existing literature focuses largely on NTM infection in the general population, specifically among immunocompromised communities, ${ }^{20,21}$ older adults, or on other more common manifestations of NTM infection in children, such as lymphadenitis, which is the most common form of NTM infection in children. ${ }^{4,15,19}$ In contrast, our study focuses on NTM pulmonary infection in infants and aims to examine existing work for relevance to infant populations, as existing literature concedes that there is very limited data "on the epidemiology, diagnosis and optimal management of nontuberculous mycobacterial (NTM) disease in children". ${ }^{15,22}$ Although findings suggest that this infection is uncommon, there has been a noted increase from 0.6 to 1.6 cases per 100,000 children per year, ${ }^{15}$ and the infection in immunocompetent individuals continues to rise. ${ }^{23}$ In relation to children, there is still limited data as many reports have fewer than 50 cases and lack details about diagnostic workup, management, and outcome. ${ }^{15}$ Finally, another factor that impedes detailed study of this infection is the fact that reporting for NTM infections is not mandatory, unlike with $M$. tuberculosis infection, hampering a full understanding of NTM infections' impact on public health. $^{24}$

Identifying more infection cases is a valuable step toward systematizing data about this disease, especially given the fact that NTM infection rates appear to be rising,,${ }^{17,25}$ although large scale studies are especially limited in pediatric or specifically infant populations. ${ }^{15}$ The median age of diagnosis in existing studies of NTM in the population is far older than infancy, as infections are most common in older adults. ${ }^{7-11} \mathrm{An}$ estimate by Diel et al found that the five-year all-cause mortality rate of patients with MAC-PD was $27 \%$ and that the pooled estimate of the one-year mortality rate was $10 \%(95 \%$ CI, 5-20\%). ${ }^{10,14}$ Patients with comorbidities (eg coexisting lung disease) and the presence of fibrocavitary disease have a higher chance of mortality. ${ }^{14}$ Our study found 2 deaths, although neither case reported comorbidities or immunodeficiencies.

Differentiating pulmonary NTM infection from tuberculosis infection remains challenging at diagnosis but is important to study, as the treatment path for tuberculosis is ineffective against NTM. ${ }^{26}$ In a 2012 study by Maiga et al, ${ }^{6} 18 \%$ of cases diagnosed as chronic tuberculosis were found to be a misdiagnosis of a solely NTM infection; a 2015 study by Shahraki et $\mathrm{al}^{27}$ isolated NTM strains in $30 \%$ of suspected multidrug-resistant tuberculosis cases. This, coupled with the potential for comorbidities between NTM infection and tuberculosis, is indicative of the importance of detection of NTM. Unfortunately, diagnosis and treatment of NTM are especially difficult in countries where tuberculosis is endemic due to a lack of resources to test for and culture NTM, as well as limited availability of drugs useful for treating NTM. Of particular note is the potential that, although true incidence of NTM pulmonary infection may be similar to tuberculosis numbers, the true prevalence of NTM pulmonary infections may be much higher due to the difficulty of curing these infections relative to tuberculosis. ${ }^{28} \mathrm{It}$ is additionally relevant to consider complications in NTM infection data due to TB burden. Associations between increased NTM disease incidence and decreased tuberculosis incidence have been reported, but this 
relationship has not been demonstrated to be causal and is based on adult populations only. ${ }^{26}$ Positive sputum samples may be likely to be misinterpreted as tuberculosis in countries with high TB burden, rendering it difficult to ascertain differences in occurrence from the existing data.

Even in settings where diagnostic tools are more readily available, NTM can pose difficulties during diagnosis because they are commonly found in the environment and are typically regarded as commensal within the human body assuming an immunocompetent host. ${ }^{26}$ Interestingly, the majority of cases our study examined were in immunocompetent children, which may support identified trends of increasing NTM infection in immunocompetent hosts. ${ }^{23}$ Recommendations for the diagnosis of NTM from the American Thoracic Society require positive culture results, in addition to radiological findings and the exclusion of other pulmonary diseases like tuberculosis. ${ }^{18}$ This is due in large part to the ubiquity of NTM in the environment and within the human body; positive culture of NTM alone is not sufficient evidence of pathogenic NTM. Gastric aspirate samples, for example, are commonly used to test for tuberculosis ${ }^{29}$ and were collected in a third of the cases in our study as well to test for NTM in culture. However, this type of testing does not conclusively prove NTM to be the pathogen, as NTM exist in the gastrointestinal tract as commensal bacteria. ${ }^{26}$

A 2016 study by Tebruegge et al $^{15}$ cited sample culture and acid-fast bacilli staining as the most sensitive diagnostic tests sensitive for NTM lymphadenitis, with $67.2 \%$ and $35.7 \%$ positivity rates respectively. Ninety-two percent of cases then tested positive using polymerase chain reaction testing for species identification. However, molecular testing methods may not be readily available or widely used at present: PCR was used in $13.9 \%$ of cases included in our study, while other techniques such as culture from bronchoscopy samples or acid-fast staining were utilized in the majority of cases. Other literature on pulmonary NTM pulmonary infection diagnosis similarly relied on or recommended acid-fast smears/staining and mycobacterial cultures as key procedures. ${ }^{30,31}$

These methods have their own limitations, however. Mycobacterial growth in culture may be attributable to sample contamination due to the prevalence of NTM in the environment, while acid-fast testing cannot differentiate between $M$. tuberculosis and other NTM strains. Additionally, acid-fast testing is limited by its variable sensitivity; ${ }^{32}$ this concern is evident in our study, where 11 out of 32 NTM cases assessed for AFB via smear or culture did not return positive results.
Although we compare our findings to existing literature, limitations within our data should also be noted. Our study looked at a small, specific population of patients, limiting us to just 37 cases. A larger data set could demonstrate that trends identified are not reflective of overall characteristics amongst the infant population. Additionally, the retrospective nature of our study relies upon the information contained within the case reports we examined, limiting our data to information that the authors of each case studies deemed relevant to report. Not all case reports were published following the conclusion of treatment, resulting in a possible reporting bias for later complications or changes in treatment regimen. Our search scope was also limited to English language published cases, leaving potential missed data in grey literature or other unpublished cases we did not identify, as well as in non-English literature. Our study also did not include formal statistical bias analysis, which is an additional drawback, though we did not detect that bias was otherwise present in the studies analyzed.

\section{Conclusion}

NTM pulmonary infection is increasing in incidence and prevalence. The characteristics of this disease in children is less well studied than in adult populations, and limited clinical guidelines drawn from patient data exist. As a result, it is important to summarize the existing literature in order to highlight the need for further research into this area.

This study compiled data from existing case reports on infants diagnosed with nontuberculous mycobacterial pulmonary infection. Our findings indicate that the most common NTM strain type is the $M$. avium complex and caused most infections in cases we examined, which supports other existing findings. Most cases opted for antibiotic treatment to combat infection, with the most commonly prescribed drugs varying for different strains of NTM. Diagnostic practices frequently involved a direct sample for culture via bronchoscopy or thoracoscopy, as well as acid-fast bacilli testing. As NTM infection rates continue to rise worldwide, it is important to highlight what data currently exists, as well as its limitations to direct additional research and data collection in order to grow the base of evidence from which clinicians can make conclusions about the course of treatment for this disease, both in the general population as well as specifically amongst children and infants. The diagnosis of infant NTM may be complicated by technical issues related to specimen collection and age limitations. Infants with NTM may be underdiagnosed as a result and our study highlights the salient findings and treatment of this infection among infants. To our knowledge, our study is the 
first to specifically examine details of NTM pulmonary infection specifically among infants.

\section{Abbreviations}

NTM, nontuberculous mycobacterial; AFB, acid-fast bacilli; MAC, M. avium complex.

\section{Acknowledgments}

The authors would like to thank Ms. Lisa Naidoo, Health Information Science, RCHSD.org for their expertise and assistance in this project. The views expressed herein are those of the authors and do not necessarily reflect the views of Brown University or Rady Children's Hospital.

\section{Disclosure}

Dr Dosanjh is an affiliated staff member of Department of Pediatrics, Rady Children's Hospital, San Diego, CA, USA. The authors have disclosed no other conflicts of interest and financial relationships relevant to this article. This review does not contain a discussion of an unapproved/investigative use of a commercial product/device.

\section{References}

1. Glatstein M, Scolnik D, Bensira L, Domany KA, Shah M, Vala S. Lung abscess due to non-tuberculous, non-Mycobacterium fortuitum in a neonate. Pediatr Pulmonol. 2012;47(10):1034-1037. doi:10.1002/ ppul.22558

2. Mirsaeidi M, Farnia P, Sadikot R, Hsueh P-R, Aliberti S. Nontuberculous mycobacteria: epidemiologic, mycobacteriologic, and clinical aspects. Bio Med Res Int. 2015;2015:523697. doi:10.1155/ 2015/523697

3. Ryu YJ, Koh W-J, Daley CL. Diagnosis and treatment of nontuberculous mycobacterial lung disease: clinicians' perspectives. Tuberc Respir Dis. 2016;79(2):74-84. doi:10.4046/trd.2016.79.2.74

4. Cruz AT, Starke JR. Nontuberculous mycobacterial disease in pediatric populations. In: Griffith DE, editor. Nontuberculous Mycobacterial Disease: A Comprehensive Approach to Diagnosis and Management. Respiratory Medicine. Springer International Publishing; 2019:439-463. doi:10.1007/978-3-319-93473-0_16

5. Osorio A, Kessler RM, Guruprasad H, Isaacson G. Isolated intrathoracic presentation of Mycobacterium avium complex in an immunocompetent child. Pediatr Radiol. 2001;31(12):848-851. doi:10.1007/ s002470100003

6. Maiga M, Siddiqui S, Diallo S, et al. Failure to recognize nontuberculous mycobacteria leads to misdiagnosis of chronic pulmonary tuberculosis. PLoS One. 2012;7(5):e36902. doi:10.1371/journal.pone.0036902

7. Cassidy PM, Hedberg K, Saulson A, McNelly E, Winthrop KL. Nontuberculous mycobacterial disease prevalence and risk factors: a changing epidemiology. Clin Infect Dis off Publ Infect Dis Soc Am. 2009;49(12):e124-e129. doi:10.1086/648443

8. Eliseev P, Hinderaker SG, Heldal E, Tarasova I, Grjibovski A, Mariandyshev A. Diagnosis and treatment of patients with pulmonary nontuberculous mycobacterial diseases in Arkhangelsk, Russia. Infect Genet Evol J Mol Epidemiol Evol Genet Infect Dis. 2019;73:358-361. doi:10.1016/j.meegid.2019.05.022
9. Henkle E, Hedberg K, Schafer S, Novosad S, Winthrop KL. Population-based incidence of pulmonary nontuberculous mycobacterial disease in Oregon 2007 to 2012. Ann Am Thorac Soc. 2015;12 (5):642-647. doi:10.1513/AnnalsATS.201412-559OC

10. Park SC, Kang MJ, Han $\mathrm{CH}$, et al. Prevalence, incidence, and mortality of nontuberculous mycobacterial infection in Korea: a nationwide population-based study. BMC Pulm Med. 2019;19 (1):140. doi:10.1186/s12890-019-0901-z

11. Prevots DR, Marras TK. Epidemiology of human pulmonary infection with nontuberculous mycobacteria: a review. Clin Chest Med. 2015;36(1):13-34. doi:10.1016/j.ccm.2014.10.002

12. Marras TK, Prevots DR, Jamieson FB, Winthrop KL; Pulmonary MAC Outcomes Group. Variable agreement among experts regarding Mycobacterium avium complex lung disease. Respirol Carlton Vic. 2015;20(2):348-351. doi:10.1111/resp. 12440

13. Daley CL, Iaccarino JM, Lange C, et al. Treatment of nontuberculous mycobacterial pulmonary disease: an official ATS/ERS/ESCMID/ IDSA clinical practice guideline. Clin Infect Dis. 2020;71(4):e1e36. doi:10.1093/cid/ciaa241

14. Diel R, Lipman M, Hoefsloot W. High mortality in patients with Mycobacterium avium complex lung disease: a systematic review. BMC Infect Dis. 2018;18(1):206. doi:10.1186/s12879-018-3113-x

15. Tebruegge M, Pantazidou A, MacGregor D, et al. Nontuberculous mycobacterial disease in children - epidemiology, diagnosis \& management at a tertiary center. PLoS One. 2016;11(1):e0147513. doi:10.1371/journal.pone.0147513

16. Park Y, Lee EH, Jung I, Park G, Kang YA. Clinical characteristics and treatment outcomes of patients with macrolide-resistant Mycobacterium avium complex pulmonary disease: a systematic review and meta-analysis. Respir Res. 2019;20(1):286. doi:10.1186/ s12931-019-1258-9

17. Piersimoni C, Scarparo C. Pulmonary infections associated with non-tuberculous mycobacteria in immunocompetent patients. Lancet Infect Dis. 2008;8(5):323-334. doi:10.1016/S1473-3099(08)70100-2

18. Waters V, Ratjen F. Antibiotic treatment for nontuberculous mycobacteria lung infection in people with cystic fibrosis. Cochrane Database Syst Rev. 2020;6:CD010004. doi:10.1002/14651858.CD010004.pub5

19. Zimmermann P, Tebruegge M, Curtis N, Ritz N. The management of non-tuberculous cervicofacial lymphadenitis in children: a systematic review and meta-analysis. $J$ Infect. 2015;71(1):9-18. doi:10.1016/j. jinf.2015.02.010

20. Martiniano SL, Nick JA, Daley CL. Nontuberculous mycobacterial infections in cystic fibrosis. Thorac Surg Clin. 2019;29(1):95-108. doi:10.1016/j.thorsurg.2018.09.008

21. Mortaz E, Moloudizargari M, Varahram M, et al. What immunological defects predispose to non-tuberculosis mycobacterial infections? Iran J Allergy Asthma Immunol. 2018;17(2):100-109.

22. Jones MM, Winthrop KL, Nelson SD, et al. Epidemiology of nontuberculous mycobacterial infections in the U.S. Veterans Health Administration. PLoS One. 2018;13(6):e0197976. doi:10.1371/journal.pone.0197976

23. Lagune M, Petit C, Sotomayor FV, et al. Conserved and specialized functions of type VII secretion systems in non-tuberculous mycobacteria. Microbiology. 2021;167(7):001054. doi:10.1099/ mic. 0.001054

24. Faria S, Joao I, Jordao L. General overview on nontuberculous mycobacteria, biofilms, and human infection. $J$ Pathog. 2015;2015:809014. doi:10.1155/2015/809014

25. Adjemian J, Olivier KN, Seitz AE, Holland SM, Prevots DR. Prevalence of nontuberculous mycobacterial lung disease in U.S. Medicare beneficiaries. Am J Respir Crit Care Med. 2012;185 (8):881-886. doi:10.1164/rccm.201111-2016OC

26. López-Varela E, García-Basteiro AL, Santiago B, Wagner D, van Ingen J, Kampmann B. Non-tuberculous mycobacteria in children: muddying the waters of tuberculosis diagnosis. Lancet Respir Med. 2015;3(3):244-256. doi:10.1016/S2213-2600(15)00062-4 
27. Shahraki AH, Heidarieh P, Bostanabad SZ, et al. "Multidrug-resistant tuberculosis" may be nontuberculous mycobacteria. Eur $J$ Intern Med. 2015;26(4):279-284. doi:10.1016/j.ejim.2015.03.001

28. Iseman MD, Marras TK. The importance of nontuberculous mycobacterial lung disease. Am J Respir Crit Care Med. 2008;178 (10):999-1000. doi:10.1164/rccm.200808-1258ED

29. Pomputius WF, Rost J, Dennehy PH, Carter EJ. Standardization of gastric aspirate technique improves yield in the diagnosis of tuberculosis in children. Pediatr Infect Dis J. 1997;16(2):222-226. doi:10.1097/00006454-199702000-00011
30. Forbes BA, Hall GS, Miller MB, et al. Practice guidelines for clinical microbiology laboratories: mycobacteria. Clin Microbiol Rev. 2018;31(2). doi:10.1128/CMR.00038-17

31. Martin A, Colmant A, Verroken A, Rodriguez-Villalobos H. Laboratory diagnosis of nontuberculous mycobacteria in a Belgium Hospital. Int J Mycobacteriol. 2019;8(2):157-161. doi:10.4103/ijmy.jjmy_40_19

32. Ryan GJ, Shapiro HM, Lenaerts AJ. Improving acid-fast fluorescent staining for the detection of mycobacteria using a new nucleic acid staining approach. Tuberc Edinb Scotl. 2014;94(5):511-518. doi:10.1016/j.tube.2014.07.004

\section{Publish your work in this journal}

Pediatric Health, Medicine and Therapeutics is an international, peerreviewed, open access journal publishing original research, reports, editorials, reviews and commentaries. All aspects of health maintenance, preventative measures and disease treatment interventions are addressed within the journal. Practitioners from all disciplines are invited to submit their work as well as healthcare researchers and patient support groups. The manuscript management system is completely online and includes a very quick and fair peer-review system. Visit http://www.dovepress.com/testimonials.php to read real quotes from published authors. 